\title{
A literature review of recent topics in Korean Military Art and Science
}

\author{
Jonggil Lee*
}

The Republic of Korea, which is the only divided country in the world, is an extremely prominent country facing an existential enemy named "North Korea". In Korea, the military science field is gaining interest and importance. However, as it is a specialized field, research is scarce. In this study, through text mining, we attempted to analyze 69 regular papers' abstracts in the military science and domestic social science fields. These abstracts were present in the Korea Citation Index $(\mathrm{KCl})$, which publishes the latest research trends and, in this case, trends in military studies. As a result, research is being conducted on various subjects. In particular, 63\% (44) of these were found to be related to the construction of military power such as security cooperation, external situations, research and development, and defense reform. This study applied an unstructured data analysis method called text mining and attempted to analyze the subject of several papers from a more scientific perspective. Hence, it can be considered a study of great significance as it was able to provide an overall understanding of general research trends and trends in recent military studies.

Keywords : military art and science, military force building and development, military force management, acquisition of weapon systems and operation, text mining, Latent Dirichlet Allocation

(a) (1) This work is licensed under a Creative Commons Attribution 4.0 International License.

* (First Author) ROK (Republic of Korea) Army Training and Doctrine Command, Major, rokleejg@gmail.com 


\section{I. 서론}

최근 한반도 주변 정세는 전통적인 북한의 위협 외에도 여러 주변국가 간의 분쟁과 대립으로 인 해 안보상 불확실성이 높아지고 있어 국방외교 차원에서 국방 및 방위력증강을 위한 노력이 더욱 중요해지고 있다. 그리고 국가 간 상호이해관계에 따른 안보강화를 위해 군사협력을 강화하고 있어 대내외 정세 변화를 이해하고 전략적 대응방안을 모색해야 한다. 이런 이유에서 "정치, 경제, 역사, 사회과학 외에 군사사상, 군사사, 군사전략 등의 고유 연구영역을 포괄하는 통섭(consilience)의 학 문”(Kil, 2012)인 군사학 분야에 대한 연구동향 탐색을 통해 전반적인 연구경향을 이해할 수 있다. 아래 Table 1은 최근 국방 / 방위 관련 뉴스이며 국방 / 방위 관련 다양한 이슈들이 존재하고 있음 을 알 수 있다. 이러한 뉴스나 기사를 통해 다양한 대외 정세변화에 따른 국가 간 안보협력, 분쟁 해결 등이 중요해지고 나아가 군사학 분야의 학문적 연구주제로 선정되고 있다. 따라서 이러한 연 구동향이나 경향을 Text Mining으로 분석하는 것은 주요한 의미를 가질 수 있을 것이다.

$<$ Table 1> Recent national defense news in Korea

\begin{tabular}{|c|}
\hline 최근 국방 / 방위 관련 뉴스 \\
\hline 美, 인도와 외교•군사협력 확대 $\cdots$ 對中 압박 강화 (문화일보, '20. 10. 26) \\
\hline “미·일·인도·호주 내달 해상합동훈련, 중국에 악몽 될 것” (한국경제, "20. 10. 22) \\
\hline 중국 견제’ 쿼드 합동군사훈련 $\cdots$ 한국에 손 내미는 美・中 (KBS NEWS, '20. 10. 21) \\
\hline ‘탈(脫)미국’ 중국 러시아와 ‘제한적 밀월’, 산업협력·군사기술 공유 (뉴스핌, ‘20. 9. 2) \\
\hline 미국이 남중국해에 정찰기 띄우자 중국 탄도미사일로 맞대응 (한겨레, '20. 8. 27) \\
\hline
\end{tabular}

본 연구는 Word Cloud분석을 통해 분석대상이 되는 군사학 논문들에서 자주 언급되는 단어들에 대해 시각화 및 정리하였으며 상위 20개의 단어를 제시하였다. 또한 LDA Topic Modeling을 통해 몇 개의 주제로 연구들을 분류하였다. 마지막으로 급변하는 국제 정세에 따른 최근 연구주제 분야 를 선행연구인 Bae et al. (2020)의 연구결과를 토대로 분류하고 주제별 연구내용을 살펴보고, 동향 을 탐색하고자 하였다. LDA Topic Modeling을 사용하여 군사학 관련 정규 논문들을 몇 개의 주제 로 분류하여 전반적인 군사학 연구의 경향과 동향을 분석하고자 하였으며 Bae et al. (2020)의 연구 에서 제시한 주제분류를 활용하여 분석의 대상이 되는 논문들이 주제별로 어떻게 분류되고 있는지 를 확인하고자 하였다. 두 가지 방법 모두 LDA Topic Modeling을 기반으로 분석하는 것이지만 첫 번째는 우선 전반적인 연구경향을 분석하기 위한 목적으로 사용되었으며 두 번째 Bae et al. (2020) 의 연구를 바탕으로 분류한 주제별로 다시 분석한 것은 기존 연구에서 분류한 주제별로 어떠한 논 문들이 포함되어 있으며 어떠한 연구들이 진행되었는지를 살펴보는 것으로 첫 번째 경향분석에서 임의 개수의 주제로 분류하는 것과는 차이가 있을 수 있다. 또한 Bae et al. (2020)의 연구는 다수의 
논문을 분류하는 방법과 주제를 제시하였다면 본 논문에서는 이러한 방법을 적용하여 전반적인 연 구의 경향과 동향을 분석하는데 목적이 있다. 본 연구에서는 이러한 선행연구의 분류법을 적용하여 군사학 논문들을 분류, 연구동향을 분석하고자 하였으며 이러한 방법은 의사결정자가 직면하는 새 로운 결정사항에 관한 시기적절한 근거를 제공할 수 있는 신속한 체계적 문헌고찰(rapid systematic review)(Schünemann \& Moja, 2015) 접근을 토대로 최신 연구주제의 특징을 분석하는 방법으로 전반적인 군사학 연구의 최신 경향 및 흐름을 이해하고 이를 토대로 미래 군사학 연구의 방향에 대한 전망을 알아보는데 적절한 연구방법이라 판단하였다.

\section{ㅍ. 연구 방법}

\section{1 선행연구 고찰}

본 연구에서는 대상이 되는 '20년도 군사학 논문 69편에 대한 Text Mining분석을 통해 최근의 연구동향과 경향, 그리고 이를 통한 군사학 연구의 미래 등을 제시하고자 연구를 진행하게 되었다. 또한 Text Mining 분석 기법 중 LDA Topic Modeling을 통해 군사학 논문들을 몇 가지 주제로 분류하고자 하였으며 마지막으로 기존 선행연구에서 제시된 군사학 논문들의 주제 분류를 활용하 여 다시 69개의 논문들을 분류해 봄으로써 연구 동향 등에 대해 추가적인 분석을 실시하고자 하 였다.

\subsubsection{Text Mining}

Kim, Cho, \& Kang (2016)의 연구자료를 참고하면 텍스트 데이터는 비정형화된 데이터의 대표 적인 예이다. 일반적으로 활용의 목적으로 가공되어지거나 별도로 수집되어지는 정형 데이터와는 다르게 비정형 데이터는 여러 가지 경로를 통해 다양한 형태로 수집, 획득, 저장되어 진다. 이렇게 수집된 비정형데이터는 과거에는 적절히 분석할 도구가 없었으나 최근에는 텍스트 마이닝 분석기 법을 통해 다양한 형태로 분석이 가능해지게 되었다. 텍스트 마이닝의 시작은 분석할 대상이 되는 텍스트 데이터들을 단어수준으로 분해하는 것이다. 본 연구에서는 분석의 대상이 되는 69개의 논문 을 대상으로 Word Cloud를 통해 단어의 빈도수에 따른 특징을 살펴볼 것이며 이어서 LDA Topic Modeling을 통해 몇 가지 주제로 분류해 볼 예정이다. 분석에 사용된 도구는 상용 분석도구인 Netminer 4.4.3.b 버전을 사용하여 Word Cloud 및 LDA Topic Modeling을 진행하였다. Netminer 는 사회관계망(SNS)을 분석하는 도구로 개발되었으나 최근에는 다양한 Semantic 분석이 가능한 패키지들이 제공되어 Text mining 분석도 가능하게 되었다. 국산 상용도구인 점에서 한글을 분석 
하는데 보다 용이할 것이라는 판단에서 본 도구를 사용하게 되었다.

\subsection{2 신속한 체계적 문헌고찰(rapid systematic review)}

체계적 문헌고찰은 연구주제와 관련된 다양한 문헌에 대해 여러 가지 시각으로 분석하고 검토하 여 명확하게 연구주제를 만들어내는 과정을 핵심으로 하고 있다. 이런 체계적 문헌고찰은 문헌을 어떻게 정리하고 요약하는지에 따라 질적(Qualitative) 고찰, 양적(Quantitative) 고찰, 메타분석 (Meta-analysis)으로 나눌 수 있다. 이러한 체계적 문헌고찰은 다양한 문헌에 대한 과학적 접근과 분석을 가능하게 해준다는 장점이 있지만, 일반적으로 매우 복잡한 과정을 거치는 것으로 알려져 있다. 이러한 단점을 극복하고자 신속한 체계적 문헌고찰(Rapid Systematic Reviews) 방법 (Schünemann \& Moja, 2015)을 적용하여 보다 포괄적이고 신속하게 연구를 진행할 수 있다. 이러 한 신속한 체계적 문헌고찰은 주제나 상황에 따라 제한적 문헌검색(제한된 원천에서 특정 범위 내 에서 제한적으로 문헌을 검색), 비뚤림 위험 평가 생략(문헌연구의 위험성에 대한 평가 생략), 좁은 연구 질문 선정(가장 필요한 연구주제에 대해 집중하여 분석)의 세 가지 방법을 적용할 수 있으며 본 연구에서는 군사학 연구 주제를 분석하기 위해 제한적 문헌검색 방법을 적용하여 연구를 진행 하였다.

\section{1 .3 군사학 연구 주제 분류}

Bae et al. (2020)은 LDA Topic Modeling을 적용하여 다양한 군사학 연구들을 주제별로 분류하 는 연구를 진행하였다. 본 연구는 군사학 연구라는 특정 분야의 학문분야에 대한 연구주제를 세분 화하여 기존 학문분류와의 상호 관련성을 탐색하는데 목적을 두고 연구를 진행하였다. 연구결과 총 10 개의 주제로 군사학 연구들을 분류하였다. 무기체계 획득/운영에서는 군사력 건설 분야에 4 가지 소분류를 하였으며 무형 군사력 유지에는 한 가지 주제를 분류하였다. 또한, 국방 연구개발 분야에 는 군사력 운용, 군사력 개발, 유형 군사력 유지 분야로 각각 1 개, 3 개, 1 개의 주제를 분류하였다. 이러한 연구결과는 군사학 연구를 몇 가지의 학문분류와 연계하여 소분류로 분류하였다는데 큰 의 의가 있는 연구라 할 수 있다. 본 연구에서는 신속한 체계적 문헌고찰을 진행하면서 Bae et al. (2020)의 연구를 바탕으로 해당 범위 내의 논문들에 대해 분석하였으며 주제를 분류하여 군사학 연 구 동향을 분석하고자 하였다.

\section{2 연구의 대상}

군사학 부문의 최근 연구동향을 탐색하기 위해 국내 군사학 분야의 학술지 정보를 한국학술지 
인용색인(Korea Citation Index)에서 온라인상으로 자료를 수집하였다. 또한, 분석의 일관성 및 목 적을 달성하기 위해 국문초록을 분석의 대상으로 선정하였다. 일반적으로 초록은 연구의 내용에 대 한 배경 및 과정, 결론을 포함하고 있기 때문이다. 특히, 군사학 분야에 국한된 학술논문만 취합하 기 위해 연구재단의 학술주제분류를 참고하여 연구대상을 대분류(사회과학)와 중분류(군사학)로 구분하여 논문을 검색하였다. 또한, 최근 2020년 게재된 정규논문(학술발표 제외)만을 선택하고 내 용 검토를 위해 원문 파일이 제공되지 않은 논문은 개별적인 학술 웹사이트를 통해 수집하였다. 최 종 수집된 논문현황을 살펴보면, 한국연구재단 등재지로 한국군사학논집(화랑대연구소 발행)에서 32 편, 한일군사문화연구(한일군사문화학회 발행) 17 편으로 나타났으며, 등재후보지로 한국군사(한 국군사문제연구원) 7편, 한국방위산업학회지(한국방위산업학회) 7편, 선진국방연구(광운대학교 방 위사업연구소) 6 편으로 총 69 편을 수집하였다. 대부분 선정된 학술지가 연간 2회 발간이 되며 12 월 에 발행이 되어 상대적으로 연간 논문편수가 낮게 나타났다(2019년 연간 96편 발행). 아래의 Table 2는 이렇게 수집된 연구대상 논문들을 학술지별로 분류한 표이다.

$<$ Table 2> Current researches on Military art and science (Jan. to Nov. 2020)

\begin{tabular}{c|c|c|c}
\hline Journal title & Academic subject areas & Publish Month & \# of included studies \\
\hline \hline $\begin{array}{c}\text { Korean Journal of } \\
\text { Military Art and Science }\end{array}$ & Military Studies & Jun. & \multirow{2}{*}{32} \\
\cline { 3 - 3 } Korea Journal of Military Affairs & Other Military Science & Jun. & 7 \\
\hline $\begin{array}{c}\text { Journal of the Korea Association } \\
\text { of Defense Industry Studies }\end{array}$ & Other Military Science & Jun. & 7 \\
\hline $\begin{array}{c}\text { Journal of Korean-Japanese } \\
\text { Military and Culture }\end{array}$ & Other Military Science & Apr. & 17 \\
\hline $\begin{array}{c}\text { Journal of Advances in } \\
\text { Military Studies }\end{array}$ & Other Military Science & Aug. & 6 \\
\hline Total & & & 69 \\
\hline
\end{tabular}

\section{III. 분석 결과}

\subsection{Word Cloud(단어 구름)}

본 연구에서는 69편의 논문을 단어 수준으로 분해하고 등장하는 단어의 빈도(Frequency)를 기준 으로 단어구름(Word Cloud)을 그려보았다. 이는 빈도수에 따라 등장하는 단어의 중요도를 쉽게 시 각화할 수 있는 장점이 있다. 아래 Figure 1은 69개의 논문을 대상으로 분석한 Word Cloud 결과이 다. 빈도수(Frequency)를 기준으로 가장 많이 언급된 상위 300 개의 단어를 대상으로 분석하였으며 
대상 단어는 명사로 한정하였다. 명사는 일반적으로 가장 핵심적인 의미를 포함하는 단어이며 주제 가 되는 단어를 포함할 가능성이 상대적으로 높은 품사로 알려져 있다. 또한, 반복적으로 자주 등장 하며 일반적으로 군사학 논문에서 많이 사용될 수밖에 없어 본 연구의 주제 추정에 문제를 야기할 수 있는 몇 개의 단어를 삭제하였으며 삭제한 단어는 다음의 Table 3과 같다(Hu, Boyd-Graber, Satinoff, \& Smith, 2014; Kintsch, 2012, p. 95).

$<$ Table 3> Unlisted words on Word Cloud

\begin{tabular}{c}
\hline 삭제 단어 목록 \\
\hline \hline 군, 군사, 연구, 분석, 결과 \\
\hline
\end{tabular}

아래 Figure 1은 이러한 Word Cloud 분석결과로 특정 단어가 매우 높게 반복되어 다양한 단어 들이 유사한 수준으로 많이 언급된 것을 알 수 있다. 특히 대한민국과 안보, 경제, 정치적으로 이해 관계가 많은 국가들과 강대국들이 다수 언급되고 있음을 확인할 수 있었다. 빈도수 상위 20개의 단 어들은 ‘전략, 일본, 북한, 한국’ 등으로 나타났다(Table 4).

많이 언급된 단어로는 전쟁, 전략, 한국, 일본, 북한, 미국, 기술, 체계, 개발 등인 것을 확인할 수 있었다. 특히 전통적 우방인 미국보다도 더 높은 수준으로 <일본>이 언급되고 있음을 확인할 수 있었다. 이는 오히려 중국보다도 높은 빈도를 보였으며 북한과 비슷한 수준임을 확인할 수 있었다. <일본>은 <전략>과 더불어 상위 2 번째 단어로 분석되었다. <북한>, <중국>, <미국> 보다도 더 빈도수가 높게 분석이 되었다. 그만큼 일본은 한반도 안보환경에서 매우 중요한 국가임을 알 수 있 었다. 특히, 미일 동맹을 중심으로 일본의 군사전략에 대한 연구는 한일, 한미일, 미일 관계와 안보

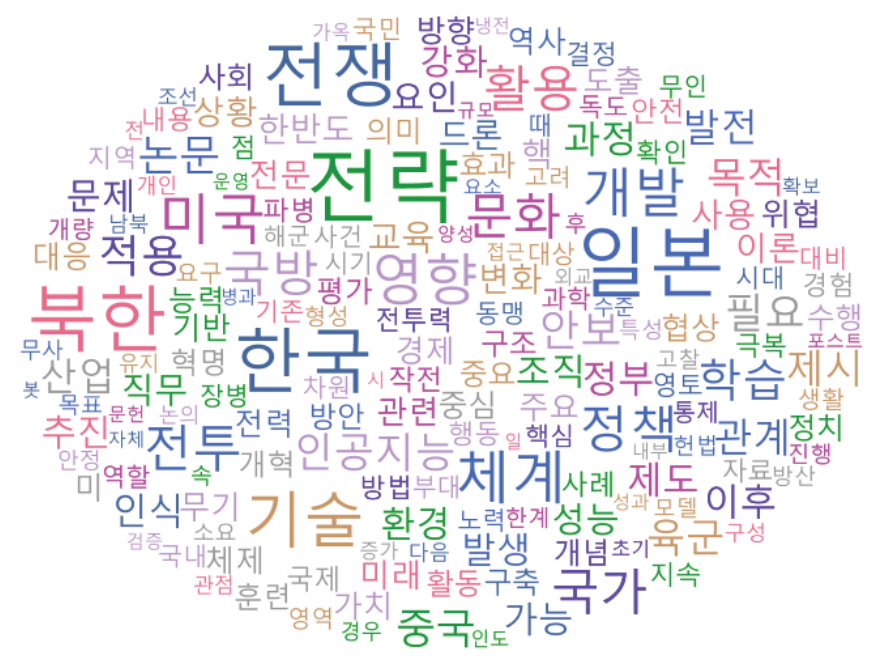

<Figure 1> Visualizing text analysis results with Word Clouds 
$<$ Table 4> Top 20 words from word frequency analysis

\begin{tabular}{c|c|c|c|c|c}
\hline Rank & Word & Frequency & Rank & Word & Frequency \\
\hline \hline 1 & 전략 & 85 & 11 & 정책 & 49 \\
\hline 2 & 일본 & 82 & 12 & 전투 & 48 \\
\hline 3 & 북한 & 75 & 13 & 활용 & 47 \\
\hline 4 & 한국 & 74 & 14 & 국방 & 47 \\
\hline 5 & 전쟁 & 74 & 15 & 문화 & 46 \\
\hline 6 & 기술 & 62 & 16 & 국가 & 46 \\
\hline 7 & 체계 & 57 & 17 & 학습 & 42 \\
\hline 8 & 영향 & 55 & 18 & 적용 & 40 \\
\hline 9 & 미국 & 54 & 19 & 안보 & 38 \\
\hline 10 & 개발 & 52 & 20 & 논문 & 38 \\
\hline
\end{tabular}

협력에 매우 중요한 문제(Kwon, 2020)라고 볼 수 있다.

또한 <기술>, <개발> 등의 단어가 높은 수준으로 언급된 것을 확인할 수도 있었다. 이는 군사학 관련 연구에서 국방기술과 무기체계의 개발 등이 매우 중요하게 다루어지는 것의 결과라 할 수 있 을 것이다. Word Cloud 분석결과 상위에 언급된 단어들은 대부분 국가와 기술, 개발, 체계 등의 단 어임을 알 수 있었다. 이러한 결과를 토대로 볼 때 대한민국을 둘러싼 다양한 안보환경에서 다양한 국가들이 영향을 주고 있음을 알 수 있었으며 또한 이러한 가운데에서도 연구의 중심에는 기술의 개발과 정책들이 중요하게 여겨지고 있음을 알 수 있었다.

\subsection{LDA Topic Modeling}

Blei et al. (2003)은 Latent Dirichlet Allocation(LDA) 알고리즘을 적용한 확률기반 주제 분류 모 델링 방법을 제안하였다. 어떠한 문서들을 특정한 주제들로 분류하기 위해 해당 주제에 속할 단어 의 확률을 추정하기 위한 알고리즘으로써 어떠한 주제에 특정 단어가 속할 확률을 추정하고 이를 통해 $n$ 개의 주제별로 해당 문서들 분류하는 알고리즘이다. 아래 Figure 2는 이러한 LDA Topic Modeling을 그래픽으로 표현한 것이다. 아래 그림에서 $\mathrm{W}$ 는 관찰된 단어들이고 $\mathrm{Z}$ 는 주제를 나타내 는 것이며 $a, \beta$ 는 코퍼스 매개변수, $\Theta$ 는 문헌의 주제 분포, $\mathrm{N}$ 은 문헌의 길이, $\mathrm{D}$ 는 문헌의 수를 각 각 의미하는 것으로, $\mathrm{LDA}$ 모델링을 통해 어떠한 단어가 특정 주제에 포함될 확률을 구하는 것이다 (Sim \& Kim, 2017). 


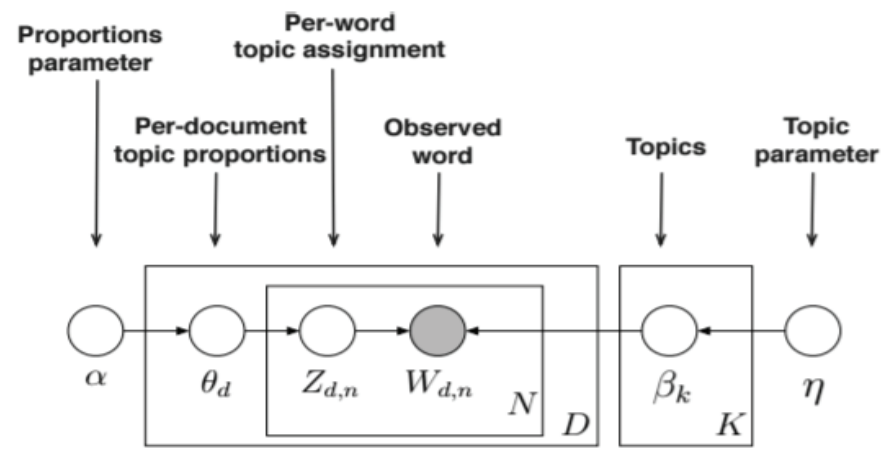

<Figure 2> LDA Topic Modeling (adopted from Blei, 2012)

본 연구의 대상이 되는 69 개의 논문을 대상으로 LDA Topic Modeling 분석방법을 적용하여 분 류한 결과는 다음의 그림과 같다. 분석의 대상이 되는 논문의 개수를 고려하여 $\mathrm{K}$ (분류할 주제의 개수) $=5$ 로 설정하였다. 본 연구에서는 분석에 사용된 문서(초록)의 수(69건)를 고려하여 3 6개의 주제로 탐색적 분석을 실시하였으며 이중 등장한 단어들이 비교적 명확하게 구분되며 상호 배타성 이 시각적으로 확인되는 주제 수 5 개를 선정하였다. 분석에는 Netminer 4.4.3.b 버전을 사용하였다.

$<$ Table 5> Top five topics in LDA topic modeling

\begin{tabular}{c|c|c|c|c|c|c}
\hline Topic \# & 1st Keyword & 2nd Keyword & 3rd Keyword & 4th Keyword & 5th Keyword & \# of documents \\
\hline \hline Topic-1 & 북한 & 정책 & 분석 & 전략 & 안보 & 19 \\
\hline Topic-2 & 기술 & 체계 & 개발 & 군사 & 적용 & 18 \\
\hline Topic-3 & 전략 & 중국 & 미국 & 군사 & 한국 & 12 \\
\hline Topic-4 & 일본 & 전쟁 & 한국 & 역사 & 자위대 & 10 \\
\hline Topic-5 & 연구 & 영향 & 활용 & 결과 & 분석 & 10 \\
\hline Total & \multicolumn{7}{|l}{} & 69 \\
\hline
\end{tabular}

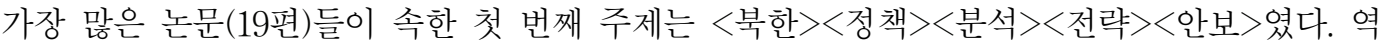
시 군사학 분야에서의 연구는 직면하고 있는 위협세력인 북한에 대한 정책과 이를 분석하고 전략 을 구축하는 안보 관련 논문들이 가장 많은 것을 확인할 수 있었다. 이는 북한의 위협은 지속되고 있으며 이를 대비하여야 하는 우리 군 고유의 특성을 잘 나타내고 있다고 볼 수 있다. 두 번째로

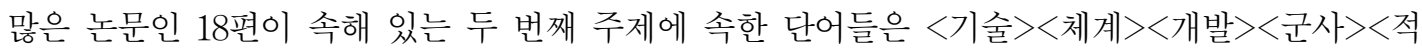
용> 이었다. 첫 번째 논문이 정책과 전략에 대한 논문들이었다면 두 번째 주제는 포함하는 단어들 로 유추해 볼 때 새로운 기술이나 체계를 개발하고 이를 군사 분야에 적용하는 것과 관련된 논문이 라는 것을 알 수 있었다. 그 다음 세 번째로 많은 논문들이 포함된 주제는 <전략><중국><미국> $<$ 군사><한국> 이었다. 세 번째 주제는 세계적인 군사강국인 중국과 미국 그리고 한국과 이들 간 
의 관계에 대한 전략에 관련된 연구임을 추정할 수 있다. 마지막 네 번째와 다섯 번째 주제는 모두 10 편의 논문이 속해 있었으며, 각각의 주제는 <일본 $><$ 전쟁 $><$ 한국 $><$ 역사 $><$ 자위대 $>$ 와 <연구> $<$ 영향 $><$ 활용 $><$ 결과 $><$ 분석 $>$ 이었다. 네 번째 주제는 이웃나라이면서 여러 가지 사안과 관련하 여 많은 대립을 하고 있는 일본에 대한 주제이다. 일본이라는 국가에 대한 역사적 관점과 자위대, 전쟁에 관한 연구가 네 번째 주제에 포함된다고 볼 수 있을 것이다. 마지막은 군사학 연구에 대한 활용이나 결과에 대한 분석, 영향에 관한 논문들이라는 것을 알 수 있었다. 이러한 LDA Topic Modeling을 통해 본 분석결과를 정리해 보면 대부분의 주제들이 한반도 주변국과 세계적인 강대국 들 사이에서의 정책과 전략에 대한 연구가 많은 것을 확인할 수 있었다. 또한 많은 논문들이 기술 이나 체계의 개발과 발전, 군사적 적용에 관한 연구였다는 것도 확인할 수 있었다.

\section{3 선행연구를 통한 Topic modeling의 군사학 학문 및 연구 분류}

대상이 되는 69개의 논문에 대해 추가적으로 군사학 학술논문 분류를 시도한 Bae et al. (2020)의 연구결과를 토대로 분류해보았다. 해당 연구는 국내 군사학 학술논문의 텍스트(초록)를 활용한 토 픽 모델링을 통해 학문분류와 연구분류표를 제시하였다. 예를 들어, 무기체계 획득 - 운영(대분류) 에 군사력 건설, 무형 군사력 유지 분야가 속하게 되며, 국방 연구개발(대분류)에 군사력 건설, 무형 군사력 유지가 포함된다. 구체적인 예시로 '군사력 건설'은 안보협력 및 대외정세 전망, 국방 - 군 개혁, 국방획득체계에서 방산원가 - 조달관리, 국방과학 연구개발 성과 및 수출경쟁력 제고 관련 세 부 연구주제를 포괄한다고 제시하였다. 해당 연계표를 참고하여 각 분류체계에 해당하는 관련 논문 을 보다 정확하게 분류하기 위해 군 경험자와 관련 분야의 주제 분류 전문가가 참여하여 논문 제 목, 주제어, 초록의 내용을 검토하여 최종적으로 분류하였다. 분류과정에서 발생하는 상이한 초기 분류를 최종적으로 동의가 될 때까지 반복하여 진행하였으며, 기존 연구에서 나타나지 않은 신규 분류항목을 별도로 추가하여 누락 없이 제시하였다. 또한, 특정 논문이 여러 소분류에 포함될 수 있는 경우에는 전체적인 연구동향을 제시하는 연구목적에 맞게 가장 대표적인 분류항목으로 구분 하였다. 전체 69 개의 논문을 내용분석을 통해 분류한다면 '군사력 건설' 부문의 안보협력 및 대외정 세 전망에 대한 연구주제가 총 24편(35\%)으로 가장 높은 빈도로 나타났다. 다음으로 '국방 군 개혁' 부문은 총 14편(20\%), 무형군사력 유지, '군 인력운영 및 군 생활 관리'는 총 9편(13\%) 수준으로 연구가 진행되었다. 아래 Table 6은 이러한 분류방식에 의해 2020년 논문의 연구주제를 분류한 표 이다.

최근 우리나라는 미국과 중국 간의 패권 경쟁이 본격화되면서 한반도 안보환경의 변화(Chung, 2017)와 기존 동맹국가(일본, 미국)과 경제적 무역마찰 등을 겪으면서 각 국가 간의 안보관계(한중, 한일 등) 유지에 어려움에 직면하고 있다. 그래서 최근 학술논문은 이런 현황을 반영하여 주변 강 대국 간의 국방외교 정책에 따른 대외정세의 변화 속에서 한반도의 안보와 국방력 강화에 초점을 
$<$ Table 6> Summary of current topics in Korean military art and science research

\begin{tabular}{|c|c|c|c|}
\hline Category & Subject & \# of studies & Reference* \\
\hline \multirow{4}{*}{$\begin{array}{l}\text { Military Force } \\
\text { Building }\end{array}$} & $\begin{array}{c}\text { Security Cooperation and } \\
\text { Current Status and } \\
\text { Prospect in military situation }\end{array}$ & 24 & $\begin{array}{l}\text { Kim, YJ; Ryu, K., \& Jeong, J.; } \\
\text { Park, J., \& Choi, K.; Park, J.; Son, K.; } \\
\text { Song, Y.; Ahn, D., \& Kil, B.; Yang, J., } \\
\text { \& Bang, J.; Lee, K.; Lee, J.; Lee, H.; } \\
\text { Jeon, J.; Choi, L.; Choi, JK.; Seo, I.; } \\
\text { Oh, S.; Song, S.; Cha, S.; Lee, S.; } \\
\text { Park, H.; Kim, JK; Kim, JC; Kim, D.; } \\
\text { Ban, K. }\end{array}$ \\
\hline & $\begin{array}{l}\text { Military Reform \& } \\
\text { Innovation }\end{array}$ & 14 & $\begin{array}{l}\text { Kwack, H., \& Kim, Y.; Kim, K.; } \\
\text { Kim, K. \& Lee, S.; Choi, K., Oh, J., \& } \\
\text { Kim, Y.; Kim, J., \& Shim, S.; Lee, H.; } \\
\text { Kim, S.; Yoon, S., \& Choi, Y.; Lee, J., } \\
\text { \& Shim, S.; Cho, S. et al.; Jang, J., \& } \\
\text { Lee, S.; Na, H., \& Choi, K.; Anais, C., } \\
\text { \& Kim, Y.; Jang, J., \& Yun, J. }\end{array}$ \\
\hline & $\begin{array}{c}\text { Defense Cost System \& } \\
\text { Government Procurement } \\
\text { management } \\
\text { in the Defense Acquisition }\end{array}$ & 4 & $\begin{array}{l}\text { Kwon, H., \& Park, J.; Na, I., \& } \\
\text { Jang, J.; Lee, J.; Choi, G. }\end{array}$ \\
\hline & $\begin{array}{l}\text { Performance of Defense } \\
\text { R\&D projects and the } \\
\text { Improvement of Export } \\
\text { Competitive Power }\end{array}$ & 2 & $\begin{array}{c}\text { Bae, H.; Lee, K., \& Han, S., \& Seol, } \\
\text { H. }\end{array}$ \\
\hline $\begin{array}{l}\text { Maintenance } \\
\text { of Intangible } \\
\text { Combat Power }\end{array}$ & $\begin{array}{l}\text { Military Human Resource } \\
\text { Management and Military } \\
\text { Life Management }\end{array}$ & 9 & $\begin{array}{l}\text { Ko, H., \& Park, J.; Lee, J.; Ku, J., } \\
\text { \& Park, H.; Kwon, H., \& Kim, Y.; } \\
\text { Cho, E., \& Lee, J.; Jeong, H., \& Ma, } \\
\text { J.; Kim, Y., \& Park, J.; Lee, H., \& } \\
\text { Kim, N.; Kim, B. }\end{array}$ \\
\hline $\begin{array}{l}\text { Military Force } \\
\text { Management }\end{array}$ & $\begin{array}{c}\text { Battle (battlefield } \\
\text { environment) Simulation }\end{array}$ & 2 & Moon, H.; Choi, J. et al. \\
\hline $\begin{array}{c}\text { Others } \\
\text { (additional } \\
\text { classification) }\end{array}$ & Military \& War History & 7 & $\begin{array}{l}\text { Kim, YH; Kim, JB; Kim, JS; Park, } \\
\text { W.; Lee, S.; Lim, H.; Jeon, G. }\end{array}$ \\
\hline $\begin{array}{l}\text { Military } \\
\text { Theory } \\
\text { (Multidisciplin } \\
\text { ary approach) }\end{array}$ & & 2 & Bae, S. et al.; Park, J. \\
\hline Unclassified & & 5 & $\begin{array}{l}\text { Gwak, S.; Kim, H.; Cho, J.; Han, Y. } \\
\text { et al.; Jeong, S., \& Kim, H. }\end{array}$ \\
\hline Total & & 69 & \\
\hline
\end{tabular}

Note. * The publication year of the paper is all 2020 . 
둔 '군사력 건설'을 주요한 주제로 다루고 있다고 볼 수 있다.

첫째, 최근 세계 안보는 권력의 전이(Power Shift)에 따른 주변 국가들의 국익 중심의 안보환경 이 조성되면서 주변국의 대외정책 및 국방정책에 대한 이해와 전략적 대응이 더욱 중요해지고 있 다. 게다가, 우리는 북한의 무력적 위협에 대비한 군사력 강화나 외교적 동맹 유지뿐만 아니라 북핵 의 문제 해결 및 통일 등의 문제해결을 위한 주변국의 협조와 협력이 필요한 실정이다(Kim \& $\mathrm{Kim}, 2014)$. 이런 의미에서 최근 학술논문은 '안보협력 및 대외정세 전망'에 대한 주제 비중이 가장 높게 나타났다. 예를 들어, 한일 관계에서 독도 영유권 분쟁(Choi, 2020), 자위대 활동문제(Son, K., 2020a) 등의 현안을 학문적 고찰을 통해 분석하여 양국 간의 안보 딜레마 상황에 보다 심층적인 이해의 필요성을 제기하였다. 한편, 한반도 평화체계 구축을 위한 안보전략을 실행하는데 관련된 주요 대상인 북한의 비핵화 추진을 위한 대한민국의 외교 및 안보정책적 방향 제시(Lee H 2020a; Jeon, J. 2020), 통일 후 미래 한국군의 조직구조 설계(Ryu \& Jeong, 2020) 등의 불확실한 안보변화 에 대응하기 위한 연구가 진행되었다. 그 외에도 다수의 논문이 이러한 국제 정세와 군사 분야에 대한 대외 이슈에 대한 연구가 진행된 것을 확인할 수 있었다.

둘째, ‘국방 · 군 개혁' 부문은 미래기술 발전에 따른 한국의 국방 및 군사전략의 변화를 추진하고 자 하는 개혁을 주제로 다루고 있다. 우리 국방부는 『2020년 국방부 업무보고』(2020. 1. 21)에서 4 차 산업혁명 기술을 적용한 스마트 국방혁신을 강조하면서 국방운영 전반의 빅데이터 구축 및 $\mathrm{AI}$ 기술 활용을 핵심과제로 설정하였다. 이런 정책추진에 부합하여 4차 산업혁명에 부합하는 무기체 계 혁신(장갑차의 원격사격통제체계: Kwack \& Kim, 2020; 무인항공기: Kim \& Shim, 2020; 인공 지능: Choi et al., 2020)과 미래전에 대응하기 위한 국방 분야의 정책적 변화방향(Kim, K., 2020; Lee, H., 2020a) 등의 최근 주제가 연구되고 있다.

셋째, '국방획득체계에서 방산원가 - 조달관리' 관련 주제는 크게 방위산업 활성화를 위한 방산수 출 증대와 방산기업의 경쟁력 강화 측면에서 연구가 진행되었다. 예를 들어, 방산수출은 “국내 방 산기업이 방산물자를 수출 시 구매국에 기술이전을 하거나, 해당 국가의 무기, 장비 또는 부품 수입 등 반대급부를 제공할 것을 조건으로 하는 교역"인 절충교역(KODIST, 방산물자교역지원센터) 활 성화 차원에서 정부 지원의 역할을 강조하고 있다(가치상계1) : Kwon \& Park, 2020). 이런 정부의 직접적인 정책적 지원 방안의 필요성 외에 국내 방산업체의 수출 경쟁력 강화를 위한 기업의 규모 경제의 중요성을 제기하거나(Choi, G., 2020) 방위산업의 지속성장가능성을 높이기 위한 방산원가 신뢰성 제고(예: 외부감사 강화, 내부통제제도 수립 등)를 통한 방위산업의 투명성 확보의 중요성 을 제시하였다(Lee, J., 2020b). 끝으로 각국 간의 미래전에 대비한 신개념 무기체계 개발과 첨단기 술 확보 활동이 증가하면서 첨단 무기에 대한 독자 개발능력이 더욱 중요해지고 있다(Gam, 2019). 특히, 방위산업 분야는 전쟁 수행에 적합한 체계개발을 위해 첨단과학기술이 활용되고 상당한 연구

1) 가치상계: 절충교역 이행방법 중 하나로 방위산업 교역에서 발생한 정부 또는 업체 간에 합의된 절충교역 의무를 상호 간에 상쇄하는 것(방위사업청, 2019, 절충교역 지침서) 
개발 비용과 장기간 개발기간이 소요된다. 그래서 국가 경제 및 방위산업체의 기업경영 활성화 차 원에서 개발 체계나 부품 등의 해외수출을 통한 내수시장 한계를 극복하는 것이 필요하다. 한편, 전 세계적으로 자국의 국방지출 예산 감소로 다국적 기업의 방산수출 확대를 위한 경쟁이 치열해 지고 있어 연구개발 기획 및 실행단계에서 수출가능성을 감안한 추진이 요구된다(Han \& Won, 2012). 이런 국가경제적 상황에 부합하여 ‘국방과학 연구개발 성과 및 수출경쟁력 제고'에 관한 연 구주제가 대두되고 있다. 특히, 급격하게 변화하는 미래전에 대비한 작전요구능력(ROC) 충족을 위 한 무기체계 개발이 요구되면서 기존 무기체계에 대한 성능개량을 통한 전력증강이 중요해지고 있 다(Bae, 2020). 또한, 우리 군이 기존에 사용하던 무기를 개량하여 해외에 수출함으로써 군의 신형 무기 보급을 확대하는데 기여할 수 있다(Kim, Y., 2018). 예를 들어, 최근 복합무기체계인 함정운용 을 통한 미래 전장 내 군사력 강화가 중요해지면서 타 무기체계와 비교하여 장기간의 획득기간과 운용기간의 특징을 지닌 함정 개발에 관련 성능개량의 중요성을 제기하였다(Bae, 2020).

다음으로 ‘무형 군사력 유지’ 관련 연구주제가 전체 논문의 약 $13 \%$ 를 차지하였다. 무형전력은 첨 단 무기체계를 운영하는 구성원의 강한 정신력으로 국방력 강화에 기여하는 주요 전투력을 의미한 다(Cho \& Park, 2016). 이런 역할 때문에 관련 연구주제는 군 장병의 인적자원관리 및 개발에 초점 을 두고 진행되었다. 예를 들어, 인적자원관리 측면에서 군 리더의 윤리적 리더십 개발(Ko \& Park, 2020), 우수 인재유인을 위한 병과별 선호도를 고려한 모집 전략방향(Lee, J., 2020a), 군 생활만족도 개선 및 직무스트레스 해소(Ku \& Park, 2020; Kwon \& Kim, 2020) 및 조직문화(구성원의 의식변 화) 개선(Cho \& Lee, 2020) 등의 구성원의 최상의 전투력 유지를 위한 인력관리 관련 주제가 연구 되었다. 그리고 인적자원개발 차원에서 미래 군 리더인 사관생도 대상의 교육 효과성(Lee \& Kim, 2020)이 연구되었다. 그 외에 또는 군 영창제도 폐지(군인사법 개정, 2020. 8. 5) 과정에 대한 법적 진행 등의 연구(Kim, B., 2020)가 진행되었다. 또한 Kim and Park (2020)은 무형전투력에 포함되어 야 하는 요소와 특징에 대해 KCTC 훈련단의 사례를 통해 분석하였다.

국방기술 연구개발은 기초연구개발, 핵심기술개발, 민-군겸용 기술개발, 신개념기술시범 (ACTD), 핵심부품군산화 개발지원이 포함되며(Defense Acquisition Program Administration, Defense Agency for Technology and Quality, Agency for Defense Development, 2015), 군사적 문 제해결을 위한 무기체계 기술의 실용화를 위한 연구나 무기체계의 핵심기술 및 부품을 개발하여 적용 - 응용하고 미래 첨단무기 독자 개발능력을 확보하는 연구방향을 의미한다. 그래서 군사력 운 용 측면에서 포병 사격에 따른 피해평가(Moon, 2020)나 비무장지대(Demilitarized zone, DMZ) 내 생태계 보존을 통한 재난 방지(산사태, 토사유실 위험) 등(Choi, 2020)의 '전투(전장환경) 시뮬레이 션 '관련 연구가 진행되었다. 또한, 미래전의 가장 위협적 형태인 사이버전은 군사적 - 비군사적 구 분이 모호하여 국가적 안보 위기를 증대할 수 있는 네트워크 중심 작전환경 $(\mathrm{NCOE}$, Network-Centric Operational Environment)(Yoon \& Choi, 2020; Lee, H., 2020b)에서 전쟁 양상이 전개될 것이다. 이런 상황에서 최근 '군사력 개발' 관련 연구는 전파방해 공격(Jamming Attack) 최 
소화 방안(Cirillo \& Kim)이나 무기체계 임베디드 소프트웨어 보증(software assurance: 신뢰성, 안 정성, 보안성)을 통한 컴퓨터 시스템의 사전적 방어능력 향상의 필요성을 제시하였다(Jang \& Yoon, 2020). 끝으로 최근 학술논문에 대한 연구주제를 탐색한 결과, 본 연구는 기존 연구의 분류체 계(Bae et al., 2020) 외에 ‘군사사 - 전쟁사', ‘군사이론'에 속하는 논문을 추가적으로 분류하였다. 예 를 들어, 관련 연구들은 대한민국의 국방 및 안보와 밀접한 역학관계인 주변 국가와의 역사적인 전쟁 - 분쟁에 대한 고찰을 통해 전쟁발생이나 전쟁실패의 원인분석을 통해 향후 군사 - 안보·외 교정책에 대한 합리적인 의사결정 방안을 제언하였다. 또한, 과거 주요 전쟁(임진왜란, 중동전쟁, 러일전쟁 등) 분석을 통해 군사전략, 전쟁수행 등의 군사력 운용이나 개발에 필요한 향후 군사혁신 방향을 제안하였다(Jeon, G., 2020). 그 외에도 Bae et al. (2020)의 연구는 군사학의 학술논문에 대 한 토픽모델링을 통해 학술적인 연구주제를 제시한 내용분석을 진행하였다.

\section{N. 논의 및 한계점}

본 연구는 국내 군사학의 최근 연구동향을 고찰하기 위해 2020년도 군사학 분야의 학술지에 게 재된 69편의 정규논문을 활용하여 내용분석을 진행하였다. 먼저 Word cloud를 통해 다수 언급된 단어들의 빈도를 시각적으로 표현하였으며 이어서 LDA Topic Modeling을 통해 임의로 5개로 주 제를 분류하였으며 다수의 논문이 속하는 주제의 핵심 단어들을 분석하였다. 이 결과 한반도 주변 을 둘러싼 강대국과 이해집단에 속하는 국가들에 대한 정책 및 전략에 대한 연구가 다수 진행되었 다는 것을 확인할 수 있었으며 더불어 기술과 체계의 개발 및 이를 통한 미래전력의 준비에 관한 연구도 다수 진행되었다는 것을 확인할 수 있었다. 마지막으로 선행연구를 통한 주제 분류에 의한 군사학 연구 주제 분류를 통해 '군사력 건설', ‘무형군사력 유지' 및 '군사사/전쟁사'의 주요 주제를 정리하였다. 이러한 연구를 통해 논의할 사항은 다음과 같다.

첫째, 변화하는 안보 및 국제정세에 따른 국내 국방 및 안보외교 대응 차원에서 '군사력 건설' 측 면의 주제가 가장 높은 비중을 차지하였다. 특히, 관련 분야의 연구들은 한반도 안보를 둘러싼 이해 관계 국가 간의 안보 및 외교 정책에 따른 안보협력과 대외정세 이해의 필요성을 중점적으로 다루 고 있다. 이런 연구동향은 북한의 핵무장 위협이나 미-중 간의 대립 등에 의한 안보정세를 이해하 고 미래 국방전략 수립을 모색하는 이론적 고찰을 제시한다고 볼 수 있다(Son, K., 2020). 게다가 향후 한반도 내 평화체계를 구축하기 위해 관련국(남한, 북한, 미국 등) 간의 역학적인 상호관계를 이해하는 접근이 더욱 중요해지고 있어 국가 간의 갈등과 협력의 상호의존 메커니즘을 심층적으로 이해해야 한다(Suh, 2008; Woo, 2003).

둘째, 국내 군사력 강화를 위한 '군 인력운영 및 군 생활관리'에 관한 '무형 군사력 유지' 영역의 
연구가 다루어졌다. 이는 군의 효율적이고 효과적인 운영과 군의 궁극적인 전쟁승리 및 군사력 강 화를 위한 장병의 정신전력의 중요성을 의미한다(Lee, 2018). 구체적으로 군 조직의 성과달성을 위 한 군 장병의 직무수행능력 강화에 필요한 직무교육과 구성원의 조직화된 전투 의지력을 높이기 위한 정신교육의 영향 $(\mathrm{Lim}, 2017)$ 과 필요성을 제기하였다. 이런 의미에서 전통적인 연구주제인 리 더십 분야 외에 최근 정보화 시대에 요구되는 직무능력 교육 차원의 온라인 교육의 효과를 다루고 있다. 셋째, '군사사/전쟁사(military/war history)' 주제는 본 연구의 내용분석을 통해 새롭게 분류 한 영역이며, 실제 전쟁 수행 방식은 전쟁의 경험을 토대로 구체적으로 개념화하고 전파할 수 있어 중요한 사례교육 내용 중 하나이다(Lee, 2019). 특히, 이런 전쟁 및 전투와 관련 기록은 당시의 군사 활동에 대한 분석을 통해 국가의 전략- 정책적 차원에서 전시 국방정책의 개발 및 수립의 토대로 활용될 수 있다(Baek, 2005).

셋째, 본 연구는 텍스트 마이닝 분석 기법을 적용하여 최근의 군사학 논문들의 연구 경향과 동향 을 분석하고자 하였다. 이러한 연구 방법을 적용한다면 군사학 연구뿐 아니라 다양한 문서, 문자로 구성된 자료에 대한 경향과 동향 분석에 대해 활용할 수 있을 것이다. 또한 다양한 뉴스와 신문기 사, 혹은 인터넷 블로그 등 문자로 구성된 다양한 형태의 수집원으로부터 수집된 자료를 분석하는 데 활용할 수 있다. 본 연구는 이러한 실무적인 사용에 활용될 수 있는 방법을 제시하고 있다는 것 도 큰 의미가 있다고 할 수 있을 것이다.

끝으로 본 연구는 군사학 분야의 문헌고찰을 통해 연구주제를 분류하는 내용분석을 진행하여 최 근 연구동향을 탐색하였다. 이런 의의에도 불구하고 연구의 제한점을 고려하여 후속연구를 위한 제 언을 다음과 제시한다. 본 연구는 신속한 체계적 문헌고찰을 통해 군사학 관련 최근 연구동향을 시 도한 초기 연구로 분석에 포함된 논문자료의 범위와 개수가 제한적이므로 향후에는 이를 보완하여 시간을 두고 체계적인 문헌고찰을 진행할 필요가 있다. 본 연구에서는 전반적인 최근 연구주제에 해당하는 토픽을 도출하였으나 향후에는 본 연구에서 도출한 각 연구영역(예: 안보, 전쟁사)을 대 상으로 주요 연구주제를 세분화하는 심층적 내용분석이 필요하다. 둘째, 현재 군사학의 연구주제에 대한 분류의 타당성을 확보하기 위해 추후 연구자들의 문헌고찰 연구가 확대될 필요가 있다. 이를 통해 연구주제 간의 상관관계와 그 의의를 보다 정확하게 이해할 수 있을 것이다.

\section{Acknowledgements}

We would like to thank Editage (www.editage.co.kr) for English language editing.

\section{Declaration of Conflicting Interests}

The author(s) declared no potential conflicts of interest with respect to the research, authorship, and/or publication of this article. 


\section{참고문헌}

Ahn, D., \& Kil, B. (2020). A Study on the Determinants of Asymmetric Force in North Korea. Korean Journal of Military Art and Science, 76(1), 249-278. https://doi.org/10.31066/kjmas.2020.76.1.010

Anais, C., \& Kim, Y. (2020). Enhanced Alternate Hop and Wait Algorithm Against A Jamming Attack in CRNs. Korean Journal of Military Art and Science, 76(2), 391-412. https://doi.org/10.31066/kjmas.2020.76.2.016

Bae, H. (2020). A Study on the Improvement of Naval Force as New Way of Force Development. Journal of the Korea Association of Defense Industry Studies, 27(1), 67-80. https://www.kci.go.kr/kciportal/ci/sereArticleSearch/ciSereArtiView.kci?sereArticleSearc hBean.artiId=ART002607190

Bae, S., \& Ku, X., \& Park, C., \& Kim, J. (2020). A Latent Topic Modeling approach for Subject Summarization of Research on the Military Art and Science in South Korea. Korean Journal of Military Art and Science, 76(2), 181-216. https://doi.org/10.31066/kjmas.2020.76.2.008

Baek, K. (2005). The Military History Compilation and Its Operating System of the ROK Army during the Korean War. Military History, 56, 165-193. UCI: G704-001528.2005..56호.005

Ban, K. (2020). The Gray Zone Strategy of Northeast Asian States and South Korea's Bespoke Counter-Measures. Korea Journal of Military Affairs, 7, 35-69. https://doi.org/10.33528/kjma.2020.6.7.35

Blei, D. M. (2012). Probabilistic topic models. Communications of the ACM, 55(4), $77-84$. https:// doi.org/10.1145/2133806.2133826

Blei, D. M., Ng, A. Y., \& Jordan, M. I. (2003). Latent dirichlet allocation. Journal of Machine Learning Research, 3(Jan), 993-1022.

Cha, S. (2020). Study on Formation of Antisocial Consciousness in North Korean Power Class: In Combination with Post-structural Theory. Journal of Korean-Japanese Military and Culture, 30, 121-146. https:/www.kci.go.kr/kciportal/ci/sereArticleSearch/ciSereArtiView.kci?sereArticleSearchBean.artiId= ART002641347

Cho, E., \& Lee, J. (2020). Tendencies and Tasks in Researches on Value of ROKA. Korean Journal of Military Art and Science, 76(2), 79-98. https://doi.org/10.31066/kjmas.2020.76.2.004 
Cho, H., \& Park, G. (2016). A Study on the Re-establishment of the Spirit Education Terms of Korean Armed Forces. Journal of Spiritual \& Mental Force Enhancement, 47, 1-56. UCI: I410-ECN-0101-2018-370-003339457.

Cho, J. (2020). War Imagery of a 'Post Memory' Novel: Focusing on Murakami Haruki's "The Wind-Up Bird Chronicle". Journal of Korean-Japanese Military and Culture, 29, 311-339. https://www.kci.go.kr/kciportal/ci/sereArticleSearch/ciSereArtiView.kci?sereArticleSearc hBean.artiId=ART002586594

Cho, S., \& Ko, J., \& Kang, S., \& Ku, X., \& Kwon, E., \& Jun, Y. (2020). A Study on Multicultural Soldiers: Development of Multicultural Receptivity Questionnaires. Korean Journal of Military Art and Science, 76(3), 315-348. https://doi.org/10.31066/kjmas.2020.76.3.012

Cho. E \& Lee, J. (2020). Tendencies and Tasks in Researches on Value of ROKA. Korean Journal of Military Art and Science, 76(2), 79-98. https://doi.org/10.31066/kjmas.2020.76.2.004

Choi, G. (2020). Study on the Direction of Defense Industry Enlargement and Integration to Strengthen Defense Industry Company Competitiveness. Journal of the Korea Association of Defense Industry Studies, 27(1), 15-28. https://www.kci.go.kr/kciportal/ci/sereArticleSearch /ciSereArtiView.kci?sereArticleSearchBean.artiId=ART002607183

Choi, J. (2020). Influence on Dokdo's Sovereignty after the Korea-Japan Agreement on Marine Border. Japanese Cultural Studies, 74, 295 - 314. https://doi.org/ 10.18075/jcs..74.202004.295

Choi, J., Shin, H., Jung, S., Kim, S., An, J., Lee, A., \& Bak, G. (2020). Selecting Native Plants for the sustainable management of the Barren Land of the Korean DMZ(Demilitarized Zone). Korean Journal of Military Art and Science, 76(2), 367-390. https://doi.org/10.31066/kjmas.2020.76.2.015

Choi, Jangkeun (2020). Japanese's Nationalism on Dokdo's Ownership and Prime Minister Yukio Hatoyama. Journal of Korean-Japanese Military and Culture, 29, 263-286. https://www.kci.go.kr/kciportal/ci/sereArticleSearch/ciSereArtiView.kci?sereArticleSearc hBean.artiId=ART002586588

Choi, K., Oh, J., \& Kim, Y. (2020). The Implications to ROK Armed Forces from the Artificial Intelligence Strategy of U.S. Department of Defense and Army. Journal of the Korea Association of Defense Industry Studies, 27(1), 41-52. https://www.kci.go.kr/kciportal/ci/sereArticleSearch/ciSereArtiView.kci?sereArticle SearchBean.artiId=ART002607187

Choi, L. (2020). Analysis on the Combined Forces of Asymmetric Relations: Analysis on 
Silla-Tang, Goryeo-Mongol(Yuan), and Joseon-Ming Relations. Korean Journal of Military Art and Science, 76(2), 217-234. https://doi.org/10.31066/kjmas.2020.76.2.009

Chung, J. (2017). A Contemplation on the Establishment of a Security Regime in the Korean Peninsula based on the Recent ROK-China Security Dilemma. Military Forum, 91, 19-41, UCI: I410-ECN-0102-2018-300-000707944

Cirillo, A., \& Kim, Y. (2020). Enhanced Alternate Hop and Wait Algorithm Against A Jamming Attack in CRNs. Korean Journal of Military Art and Science, 76(2), 391-411. https://doi.org/10.31066/kjmas.2020.76.2.016

Defense Acquisition Program Administration, Defense Agency for Technology and Quality, Agency for Defense Development (2015). Introduction of Defense R\&D, Seoul. https://url.kr/GPLmlJ

Gam, H. (2019). A Study on the Investment Efficiency of Defense Science and Technology R\&D. Journal of the Korea Academia-Industrial Cooperation Society, 20(11), 164-169. https://doi.org/10.5762/KAIS.2019.20.11.164

Gwak, S. (2020). An Epidemic models applied with isolation conditions for people contacted with confirmed cases. Korean Journal of Military Art and Science, 76(2), 433-452. https://doi.org/10.31066/kjmas.2020.76.2.018

Han, B., \& Won, J. (2012). Research on R\&D requirement planning support strategies to foster arms exports: focused on researching the evaluation model of marketability of weapon systems. Journal of Technology Innovation, 20(3), 93-128. UCI: G704-001004.2012.20.3.009

Han, Y., \& Choi, S., \& Lim, J., \& Lim, S. (2020). A Research on the Prediction of Outside Accident of the Troops based on Earthquake Analysis Model. Korean Journal of Military Art and Science, 76(3), 119-144. https://doi.org/10.31066/kjmas.2020.76.3.005

Hu, Y., Boyd-Graber, J., Satinoff, B., \& Smith, A. (2014). Interactive topic modeling. Machine Learning, 95(3), 423 - 469. https://doi.org/10.1007/s10994-013-5413-0

Jang, J \& Yoon, J. (2020). Cyber-deterrence in National Defense: Assurance Measures of Embedded Software in Weapon Systems. Korean Journal of Military Art and Science, 76(1), 349-373. https://doi.org/10.31066/kjmas.2020.76.1.014

Jang, J., \& Lee, S. (2020). A Coupled CNN Algorithm for the Reduction of False Positive Rate in Detecting Aircraft within Satellite Imagery. Korean Journal of Military Art and Science, 76(3), 61-90. https://doi.org/10.31066/kjmas.2020.76.3.003

Jang, J., \& Yun, J. (2020). Cyber-deterrence in National Defense: Assurance Measures of Embedded Software in Weapon Systems. Korean Journal of Military Art and Science, 76(1), 349-373. https://doi.org/10.31066/kjmas.2020.76.1.014 
Jeon, G. (2020). An Analysis of the Russo-Japanese War in Terms of Military Strategy with the Focus on the Ground Operations. Korea Journal of Military Affairs, 7, 145-176. https://doi.org/10.33528/kjma.2020.6.7.145

Jeon, J. (2020). Japan's Security Policy on the Denuclearization of North Korea. Journal of Korean-Japanese Military and Culture, 29, 5 - 29. https://doi.org/10.47563/kjmc.29.1

Jeong, H., \& Ma, J. (2020). Impact of individual factors and work satisfaction on professional identity of test and evaluators in ROKAF. Korean Journal of Military Art and Science, 76(2), 29-50. https://doi.org/10.31066/kjmas.2020.76.2.002

Jeong, S., \& Kim, H. (2020). A study on the adsorption properties of diisopropyl methylphosphonate on zirconium based metal organic frameworks. Korean Journal of Military Art and Science, 76(3), 455-474. https://doi.org/10.31066/kjmas.2020.76.3.017

Kil, B. (2012). Military Art and Science : Theoretical Review of Consilient Academic System and Normal Science. Journal of Military History Studies, 133, $245-277$. https://doi.org/10.17934/jmhs..133.201206.245

Kim, B. (2020). A Study on the Abolition Process of the Soldiers Guardhouse Disposition. Journal of Advances in Military Studies, 3(2), 47-61. https://doi.org/10.37944/jams.v3i2.57

Kim, D. (2020). Is the Indo-Pacific Strategy of U.S. Containment Strategy for China in the 21st Century?: Analysis of Threat Awareness in U.S. Strategic Documents and Comparison of Containment Strategy for the Soviet Union. Korea Journal of Military Affairs, 7, 71-112. https://doi.org/10.33528/kjma.2020.6.7.71

Kim, H. (2020). A comparative study on utilization of tourism contents using three major samurai residence town in Kagoshima prefecture. Journal of Korean-Japanese Military and Culture, 29, 287-309. https://www.kci.go.kr/kciportal/ci/sereArticleSearch/ciSereArtiView.kci?sereArticleSearchBean.artiI $\mathrm{d}=$ ART002586590

Kim, J., \& Shim, S. (2020). A Case Study on the Evolutionary Development of U.S Unmanned Aerial Vehicles (UAVs): Focusing on Tactical/Strategic Fixed-wing UAVs. Journal of Advances in Military Studies, 3(2), 17 - 46. https://doi.org/10.37944/jams.v3i2.69

Kim, Jaechul. (2020). An Evaluation of the Implementation of the 9.19 South-North Korea's Military Agreement and Arms Control Strategy on the Korean Peninsula. Korea Journal of Military Affairs, 7, 1-34. https://doi.org/10.33528/kjma.2020.6.7.1

Kim, Jeongki. (2020). A analysis for North Korea's Provocations and Responses of South Korea's Conduct of War System(COWS) during the 2010s. Korean Journal of Military Art and Science, 76(3), 349-390. https://doi.org/10.31066/kjmas.2020.76.3.013

Kim, Jinsoo. (2020). The Australian Army's Participation in the Korean War and the Battle of 
Gapyeong District. Korean Journal of Military Art and Science, 76(2), 235-258. https://doi.org/10.31066/kjmas.2020.76.2.010

Kim, Joonbae. (2020). The discourse of Yi Sun-sin in Japanese literature during the Meiji period. Journal of Korean-Japanese Military and Culture, 29, 235-261. https://www.kci.go.kr/kciportal/ci/sereArticleSearch/ciSereArtiView.kci?sereArticleSearc hBean.artild=ART002586586

Kim, K. (2020). The Fourth Industrial Revolution and the ROK's Defense Innovation Tasks. Korea Journal of Military Affairs, 7, 113-143. https://doi.org/10.33528/kjma.2020.6.7.113

Kim, K., \& Lee, S. (2020). Analysis and Evaluation of Factors Affecting the Success of Defense Reforms: Focusing on major defense reform cases since the Roh Tae-woo administration. Journal of Korean-Japanese Military and Culture, 29, 115-143. https://www.kci.go.kr/kciportal/ci/sereArticleSearch/ciSereArtiView.kci?sereArticleSearc hBean.artiId=ART002586576

Kim, S. (2020). Recognition of 'Defense Cost Sharing' through Big Data: Focusing on Twitter. Journal of Advances in Military Studies, 3(1), 51-74. https://doi.org/10.37944/jams.v3i1.64 Kim, S., Cho, H., \& Kang, J. (2016). The Status of Using Text Mining in Academic Research and Analysis Methods. Journal of Information Technology and Architecture, 13(2), 317-329. UCI: G704-SER000010357.2016.13.2.010

Kim, Y., \& Kim, K. (2014). Foreign Policy and Defense Policy of the Neighboring States and Responding Strategy of the ROK. Strategic Studies, 21(63), 5-34. UCI : G704-001467.2014.21.63.006

Kim, Y., \& Park, J. (2020). The Study on the Recognition of Important Degree by Intangible Combat Power Components. Journal of Advances in Military Studies, 3(1), 75-92. https://doi.org/10.37944/jams.v3i1.68

Kim, Yongjun. (2020). The United Nations and Nation Building of the Republic of Korea: Dodson Report and Roles of the UNKRA and UNCACK. Korean Journal of Military Art and Science, 76(2), 1-28. https://doi.org/10.31066/kjmas.2020.76.2.001

Kim, Younghwan. (2020). Revaluation of the Causes of Qing's Invasion into Joseon(1636) through the alliance and the adversary game. Korean Journal of Military Art and Science, 76(2), 51-78. https://doi.org/10.31066/kjmas.2020.76.2.003

Kim. Y. (2018). Innovation of acquisition system through the guarantee of evolutionary ROC application. Defense Technology, Jan(467), 32-37. UCI(KEPA) : I410-ECN-0101-2018-394-001674059

Kintsch, W. (2012). What kinds of language statistics must be in long-term memory to make language understanding possible. In Britt, A., Goldman, S., and Rouet, J. F. eds. 
Reading-From Words to Multiple Texts, 92-100. New York and London: Routledge. https://doi.org/10.4324/9780203131268-10

Ko, H., \& Park, J. (2020). The Effect of Commander's Ethical Leadership on Job Performance and Organizational Citizenship Behavior: Moderating Effect of LMX. Korean Journal of Military Art and Science, 76(1), 143-168. https://doi.org/10.31066/kjmas.2020.76.1.006

Ku, J., \& Park, H. (2020). A Study on the Effect of the Resilience and Military Job Satisfaction of Professional Soldier: Focused on Non-commissioned Officer of Special Forces. Review of Korean Military Studies, 9(1), 165-185. https://doi.org/10.34166/rokms.2020.9.1.165

Kwack, H., \& Kim, Y. (2020). Consideration of the necessity installing the RCWS of current tanks and armored vehicles for the 4th industrial age. Journal of Korean-Japanese Military and Culture, 29, 181 - 205. https://doi.org/10.47563/kjmc.29.7

Kwon, H., \& Kim, Y. (2020). Phenomenological study on job stress experience of junior sergean. Korean Journal of Military Art and Science, 76(1), 111-142. https://doi.org/10.31066/kjmas.2020.76.1.005

Kwon, H., \& Park, J. (2020). A Study on the Swap way of Off-set for the promotion of Export of the Defense Industry. Journal of the Korea Association of Defense Industry Studies, 27(1), 81-95. https://www.kci.go.kr/kciportal/ci/sereArticleSearch/ciSereArtiView.kci?sereArticleSearchBean. artild=ART002607191

Kwon, T. (2020). Japan's Military Strategy and Our Response. Military Forum, 100, 221-291.

Lee, C. (2018). A Study on Practical Teaching Methods for Establishing the Value of Soldiers - Focusing on Applying the Teaching Methods of the Questioning and Questions Method. Journal of Spiritual \& Mental Force Enhancement, 55(11), 137-185. UCI: I410-ECN-0101-2019-370-000030364.

Lee, H. (2020a). Motives for North Korea's nuclear weapons development and denuclearization. Korean Journal of Military Art and Science, 76(1), 1-32. https://doi.org/10.31066/kjmas.2020.76.1.001

Lee, H. (2020b). Trends of developing training in advanced countries and direction of the Army: Focusing on the LVCG training system. Journal of Advances in Military Studies, 3(2), 1-15. https://doi.org/10.37944/jams.v3i2.73

Lee, H., \& Kim, N. (2020). Exploring language learning strategies in ESP learning via a mixed-methods approach: A case of learning military English. Korean Journal of Military Art and Science, 76(2), 283-302. https://doi.org/10.31066/kjmas.2020.76.2.012

Lee, J. (2020a). A Study on the Strategy for Recruiting elite officers of the Army branches based on the Multi-Dimensional Scaling. Korean Journal of Military Art and Science, 76(1), 
33-60. https://doi.org/10.31066/kjmas.2020.76.1.002

Lee, J. (2020b). A Study on the Construction of Trust-Based Cost Calculation Process. Journal of the Korea Association of Defense Industry Studies, 27(1), 29-40. https://www.kci.go.kr/kciportal/ci/sereArticleSearch/ciSereArtiView.kci?sereArticleSearc hBean.artiId=ART002607184

Lee, J. (2020c). U.S. Occupation Policy and Yoshida regime's Policy Initiative. Journal of Korean-Japanese Military and Culture, 29, 59-83. https://www.kci.go.kr/kciportal/ci /sereArticleSearch/ciSereArtiView.kci?sereArticleSearchBean.artiId=ART002586574

Lee, J., \& Shim, S. (2020). Development of Technology-Based Requirement Planning System in Connection with Advanced Technology in the Fourth Industrial Revolution. Journal of Advances in Military Studies, 6(1), 1-22. https://doi.org/10.37944/jams.v3i1.54

Lee, K. (2020). A Study on North Korean Spies: Interviews with Former Elite Secret Agents of North Korea. Korean Journal of Military Art and Science, 76(1), 301-330. https://doi.org/10.31066/kjmas.2020.76.1.012

Lee, K., \& Han, S., \& Seol, H. (2020). US-Israel Defense R\&D Innovation Trends and Implications: Focused on the Development of Operational Test and Evaluation of Korean Armed Forces. Korean Journal of Military Art and Science, 76(3), 199-224. https://doi.org/10.31066/kjmas.2020.76.3.008

Lee, S. (2019). Seeking the figures playing the role of Karl Clausewitz and Alfred Thayer Mahan in R.A.F : The Military History Education in the R.A.F. Staff College of Great Britain during the Inter-war Period. Military History, 113, 133 - 167. https://doi.org/10.29212/mh.2019..113.133

Lee, S. (2020). A Comparative Study on the China and Japan's Geopolitical Perceptions toward Korean Peninsula during the First Sino-Japanese War: Focus on the Li Hong-Zhang and Yamagata Aritomo. Journal of Korean-Japanese Military and Culture, 30, 147-190. https://www.kci.go.kr/kciportal/ci/sereArticleSearch/ciSereArtiView.kci?sereArticleSearc hBean.artiId=ART002641352

Lee, S. (2020). A Study on the Dispatch and Activities of the Korean War Correspondent During the Vietnam War. Korean Journal of Military Art and Science, 76(2), 127-150. https://doi.org/10.31066/kjmas.2020.76.2.006

Lim, H. (2020). A Study on the Way to Remember the dead during a War: Focused on the R.O.K Airmen killed during the Korean War. Korean Journal of Military Art and Science, 76(1), 279-300. https://doi.org/10.31066/kjmas.2020.76.1.011

Lim, Y. (2017). A Study on the Directions to Increase Patriotism in the Moral Education at the ROK Armed Forces. Korean Journal of Military Affairs, 2, $187-230$. 
https://doi.org/10.33528/kjma.2017.12.2.187

Moon, H. (2020). A study on modeling of artillery probable errors for improving simulation logic of artillery damage assessment in war-game models. Korean Journal of Military Art and Science, 76(1), 331-348. https://doi.org/10.31066/kjmas.2020.76.1.013

Na, H., \& Choi, K. (2020). China's Revolution in Military Affairs based on Artificial Intelligence: Focusing on Military Intelligentization. Korean Journal of Military Art and Science, 76(3), 91-118. https://doi.org/10.31066/kjmas.2020.76.3.004

Na, I., \& Jang, J. (2020). An empirical study on the factors affecting Arms Imports : focused on the Korean arms importing countries. Journal of the Korea Association of Defense Industry Studies, 27(1), 53-66. https:/www.kci.go.kr/kciportal/ci/sereArticleSearch/ciSereArtiView.kci?sereArticleSearchBean.artiId $=$ ART002607189

Oh, S. (2020). Literary Men and Patriotic Association of Japanese Literature under the Asian/Pacific War, and Responsibility for the War. Journal of Korean-Japanese Military and Culture, 30, 191-212. https://www.kci.go.kr/kciportal/ci/sereArticleSearch/ciSereArtiView.kci?sereArticleSearchBean.a rtiId=ART002641354

Park, H. (2020). The Nuclear Balance against North Korea and South Korea-Japan Security Cooperation. Journal of Korean-Japanese Military and Culture, 30, 63-90. https://www.kci.go.kr/kciportal/ci/sereArticleSearch/ciSereArtiView.kci?sereArticleSearc hBean.artiId=ART002641341

Park, J. (2020). A Study on the Awareness of the Noncommissioned Officer's Position in the Army: A grounded theory based approach. Review of Korean Military Studies, 9(1), 55-82. https://doi.org/10.34166/rokms.2020.9.1.55

Park, J. (2020). Analysis on the Necessity and Probability of Reserved Forces Deployment. Korean Journal of Military Art and Science, 76(2), 303-340. https://doi.org/10.31066/kjmas.2020.76.2.013

Park, J., \& Choi, K. (2020). A Biological Threat of North Korea and South Korea's Preparations. Korea Journal of Military Affairs, 7, 177-207. https://doi.org/10.33528/kjma.2020.6.7.177

Park, W. (2020). The way to Ugaki reductions: the Discussion on Disarmament in the Japanese Army in the early 1920s. Journal of Korean-Japanese Military and Culture, 29, 207-234. https://www.kci.go.kr/kciportal/ci/sereArticleSearch/ciSereArtiView.kci?sereArticleSearc hBean.artiId=ART002586583

Ryu, K., \& Jeong, J. (2020). Designing Method of the Korean Military Organization Structure in the Post Unification: Applying Resource Mobilization Theory and Strategic Choice Theory. Korean Journal of Military Art and Science, 76(1), 221-248. https://doi.org/10.31066/kjmas.2020.76.1.009 
Schünemann, H. J., \& Moja, L. (2015). Reviews: Rapid! Rapid! Rapid! ‥and systematic. Systematic Reviews, 4(1). https://doi.org/10.1186/2046-4053-4-4

Seo, I. (2020). A Study on the Changes in Marine Policy of Japan-China and the Current State of Security in East Asia over Senkaku Islands Dispute. Journal of Korean-Japanese Military and Culture, 30, 91-120. https://www.kci.go.kr/kciportal/ci/sereArticleSearch/ciSereArtiView.kci?sereArticleSearc hBean.artiId=ART002641343

Sim, J., \& Kim, H. (2017). A Searching Method for Legal Case Using LDA Topic Modeling. Journal of the Institute of Electronics and Information Engineers, 54(9), $67-75$. https://doi.org/10.5573/ieie.2017.54.9.67

Son, K (2020a). JSDF's History of Existential Contradiction to Peace Constitution. Joumal of Korean-Japanese Military and Culture, 29, 31-58. https://www.kci.go.kr/kciportal/ci/sereArticleSearch /ciSereArtiView.kci?sereArticleSearchBean.artiId=ART002586573

Son, K. (2020b). The Direction of Future Defense Strategy Based on the Security Conditions Surrounding the Korean Peninsula. KRINS QUARTERLY, 5(1), $249-273$. https://doi.org/10.46322/krinsq.5.1.7

Song, S. (2020). Prospect of the US-China Relation in the Post-Coronavirus Age: Based on the Thucydides Trap. Journal of Korean-Japanese Military and Culture, 30, 5-36. https://www.kci.go.kr/kciportal/ci/sereArticleSearch/ciSereArtiView.kci?sereArticleSearc hBean.artild=ART002641318

Song, Y. (2020). The Implication of the Cases of Military Government in Korean Peninsular for Stability Operations in the Future. Korean Journal of Military Art and Science, 76(1), 169-202. https://doi.org/10.31066/kjmas.2020.76.1.007

Suh, B. (2008). The Change of Security Order on the Korean Peninsula in the post cold-war era: Focus on Strategic Triangle of North Korea, South Korea and U.S. National Strategy, 14(2), 63-85.

Woo, S. (2003). Triangle Research and Understanding Northeast Asian Politics. Asian Perspective, 33-63. UCI: G704-000297.2003.27.2.005

Yang, J., \& Bang, J. (2020). Taiwan Military Reform: Current Status, Evaluation, and Implications for the Republic of Korea. Journal of Korean-Japanese Military and Culture, 29, 145-179. https://www.kci.go.kr/kciportal/ci/sereArticleSearch/ciSereArtiView.kci?sereArticleSearchB ean.artiId=ART002586578

Yoon, S., \& Choi, Y. (2020). Development of Defense Dronebot Integrated Control System. Journal of Advances in Military Studies, 3(1), 35 - 50. https://doi.org/10.37944/jams.v3i1.65 
2020년 국방부 업무보고. http://www.korea.kr/news/pressReleaseView.do?newsId=156372173 美, 인도와 외교·군사협력 확대… 對中 압박 강화 (문화일보, 2020. 10. 26)

http://www.munhwa.com/news/view.html?no=2020102601071403013001

미국이 남중국해에 정찰기 띄우자 중국 탄도미사일로 맞대응 (한겨레, 2020. 8. 27)

http://www.hani.co.kr/arti/international/china/959632.html\#csidx0be7f3f306e833db764c1bd212e58ce 미·일·인도·호주 내달 해상합동훈련, 중국에 악몽 될 것 (한국경제, 2020. 10. 22)

https://www.hankyung.com/international/article/202010228805Y

'중국 견제' 쿼드 합동군사훈련...한국에 손 내미는 ⿱⿱䒑土大··中 (KBS NEWS, 2020. 10. 21)

https://news.kbs.co.kr/news/view.do?ncd=5030690

'탈(脫)미국' 중국 러시아와 '제한적 밀월', 산업협력·군사기술 공유 (뉴스핌, 2020. 9. 2)

https://www.newspim.com/news/view/20200902000647 


\section{군사학의 최신 연구주제 리뷰}

이종길*

대한민국은 전 세계 유일의 분단국가이며 현존하는 <북한> 이라는 전통적인 위협세력과 직면해 있는 군사 적으로 매우 중요한 국가이다. 이러한 대한민국에서 군사학 분야에 대한 관심과 중요성은 더욱 높아지고 있으 나 전문적인 영역인 만큼 소수에 의해 연구되고 있는 것이 현실이다. 본 연구에서는 이러한 군사학 연구의 최 근 연구동향과 흐름을 발간중인 한국연구재단에 제시된 국내 사회과학 분야의 군사학 영역에 해당되는 정규 논문 69건의 초록에 대해 Text Mining을 통해 분석하고자 하였다. 분석결과 다양한 주제들에 대한 연구가 진 행되고 있으며 특히 전체의 $63 \%$ (44건)의 논문들이 안보협력 및 대외정세, 연구개발, 국방 개혁 등의 군사력 건설과 관련된 주제였음을 확인할 수 있었다. 본 연구는 Text Mining이라는 비정형데이터 분석방법을 적용하 여 다수의 논문들의 주제를 보다 과학적으로 분석하고자 노력하였으며 이를 토대로 최근 군사학 연구의 동향 과 경향에 대한 전반적인 이해를 제공할 수 있었다는 점에서 큰 의의가 있는 연구라 할 수 있을 것이다.

주제어 : 군사학, 군사력 건설 및 개발, 군사력 운용, 무기체계 획득 및 운영, 텍스트마이닝, 잠재 디리클레 할당

* 육군 교육사령부 소령 
